\title{
Control Challenges in Wireless Sensor Networks
}

\author{
John A. Stankovic \\ Department of Computer Science University of Virginia, USA
}

\begin{abstract}
Wireless sensor networks (WSN) composed of large numbers of small devices that self-organize are being investigated for a wide variety of applications. Applications, such as military surveillance and large scale assisted living facilities are key examples of applications that can benefit from WSN. Current research for WSN is widespread. However, many of the proposed solutions are developed with simplifying assumptions about wireless communication and the environment, even though the realities of wireless communication and environmental sensing are well known. Many of the solutions are evaluated only by simulation. In this talk I describe a fully implemented system, called VigilNet, consisting of a suite of more than 30 synthesized protocols (40,000 lines of code). The system supports a power aware surveillance, tracking and classification application running on 203 XSM motes and evaluated in a realistic, large-area environment. Technical details and evaluations are presented for several of the key services. In developing such systems various types of control challenges occur. I will also discuss a number of these challenges, possible solutions and open challenges.
\end{abstract}

